\title{
On the role of chirality in structure-odor relationships
}

\author{
M.Chastrette, C.Rognon, P.Sauvegrain' and R.Amouroux \\ Laboratoire de Chimie Organique Physique, Université Lyon I, 43 Boulevard \\ du 11 Novembre 1918, 69622 Villeurbanne cedex, France and 'Firmenich \\ SA, Case postale 239, CH 1211, Genève 8 Suisse
}

\begin{abstract}
The influence of chirality on odors was studied on 16 enantiomeric pairs according to the dispersion/hydrogen bonding theory of receptor-odorant interaction.

Comparisons of molecular structures were made by superimposition of optimized conformations, using the Alchemy II package. The quality of fit was assessed using the RMS parameter included in Alchemy II and a new index for hydrogen bonding: the angle between $\mathrm{H}$-bonds in the two molecules.

In the case of camphoraceous odorants where an interaction model was already known superimposition according to the model led to correct predrctions of the high similarity of odors observed in enantiomeric pairs.

For several urinous odorants comparisons were made using $d$-androstenone as a reference compound for the urinous odor. Correct predictions were obtained for $l$-androstenone, both enantiomers of androsta-4, 16-dienone, and $(+)-2$-methyl-4-(5,5,6-exo-trimethyl-2-exonorbomyl)-cyclohexane. The $(-)$ enantiomer of the latter compound was correctly predicted only if it was assumed that its weak intensity is due to a partial interaction with the hydrophobic zone of the receptor.

For ambergris odorants which have a complex odor (-)-Ambrox was selected as the reference compound. The odors of ( +$)$-Ambrox and enantiomers of four other compounds (ambergris or woody) were correctly predicted by superimposition.

For nookatone and three derivatives which have a grapefruit note for one enantiomer and a woody note for the other no models or reference compounds were available. The superimpositions were made between grapefuit enantiomers, on the one hand, and woody enantiomers on the other hand. Woody and grapefruit characters were correctly predicted in all cases.

The limits of this approach based on molecular modelling are discussed.
\end{abstract}

\section{Introduction}

The concept of a receptor in which the bioactive molecule fits in order to exert its action was developed around the turn of the century. More recently, Hansch and his associates (Hansch et al., 1963; Hansch and Leo, 1979) showed that bioactivity could be quantitatively correlated with physicochemical properties. The study of enantiomers which have the same physicochemical properties, but may differ in their bioactivities offers a way to assess the relative importance of the two processes of transfer to and interaction with the sites.

Chirality plays an important role in many interactions with biological sites such as drug response (Mustchler et al., 1986; Testa, 1986), insect communication (Silverstein, 1978) or human taste (Schallenberger and Acree, 1971; Pickenhagen and BrönnerSchindler, 1984; Mosandl and Heusinger, 1985).

In spite of the difficulty of preparing enantiomerically pure compounds and of the sometimes low chiral specificity of human odor discrimination, works on interconversion of carvone enantiomers (Friedman and Miller, 1971) or selective synthesis and separation of enantiomers (Theimer et al., 1977; Beets, 1978c; Ohloff, 1986; Mosandl and Günther, 1989; Mosandl et al., 1990), electrophysiological studies on the olfactory receptor cells of the frog (Revial et al., 1982, 1983) and sensory tests (Pike et al., 1988; Polak et al., 
1989) have shown that chirality, as expected, influences the odor displayed by pure compounds.

However, there is no general rule and every kind of situation appears in enantiomeric pairs: odors can be identical, of the same quality with different intensities for each note or facet, or completely different with perhaps one odorless enantiomer.

\section{Theories of receptor-odorant interactions}

It seems that olfaction is a bimolecular process involving a reversible and non covalent interaction of an airborne molecule with a complementary site of a receptor system, which takes place at the interfaces of peripheral nerve cells located within the mucous layer of the olfactory epithelium. Recent results confirm that receptors are parts of proteins, and suggest that several hundred different types of receptors may be involved in interactions (Buck and Axel, 1991). The modes of interaction between an odorant molecule and a receptor site are still unclear, although several modes have been proposed in different theories. The most important are the stereochemical theory proposed by Amoore and Beets' functional group theory.

Amoore (1963a, 1963b, 1971) considers that two molecules can interact with the same receptor site if they have the same global shape, but he does not consider their orientations.

According to Beets $(1978 \mathrm{a}, \mathrm{b})$, the odor of a molecule is directly related not only to its shape, but also to its dipole vector, which in turn is related to the disposition of the functional group(s). Molecular flexibility is also an important parameter: reorientations and conformational adaptations to the requirements of the sites take place during the formation of the interaction complex. Consequently, the interactions between a population of structurally and configurationally identical stimulant molecules and a population of receptor sites of a chemoreceptory system generate an information pattern which can be described with a series of notes ranking from the main (the strongest ones) to secondary notes.

Beets correlates the differences of odors between enantiomers with their functionalities and their flexibilities. To quantify his results, following a known methodology (Amoore, 1964; Allinger, 1972), he compares two enantiomers by matching them in such a way that their dipolar axes are superimposed and estimates the quality of fit with a $D_{M}$ (morphological difference) parameter measuring the bulk difference between the two enantiomers [having their dipolar axes matched] (Beets, 1978a). Three kinds of molecules are distinguished: apolar ones, for which there should be little or no influence of the chirality, monofunctional polar ones, for which differences should concern mostly the intensity and the secondary notes and, lastly, polyfunctional polar molecules which should be very sensitive to chirality. This general trend has to be modulated according to the molecular flexibility which allows a conformational adaptation of the compound to the receptor site requirements (Beets, 1978b,c).

Considering that receptors are located on proteins it seems reasonable to assume that hydrogen bonding plays a determinant role in the receptor-molecule interactions. Indeed a study of structure-odor relationships for musk odorants led Chastrette and Zakarya (1988) to propose an interaction model based on both hydrogen bonding (as the main type of interaction) and dispersion forces. A good fit between the corresponding parts of the molecule and of the receptor site appeared to be a condition for the recognition 
of an odorant and consequently the geometrical requirements of the interaction are key factors. The model for the musk odor of nitro aromatic musks (Chastrette and Zakarya, 1988; Zakarya, 1988) consists of a $t$-butyl group which interacts with the hydrophobic part of the receptor site through dispersion forces, and a functional group (nitro or carbonyle) which interacts with the hydrophilic part of the receptor site through hydrogen bonding (Chastrette, 1990; Zakarya, 1988). The hydrogen atom of the hydrogen bond and the quaternary carbon in the $t$-butyl group must be $5 \dot{\mathrm{A}}$ apart.

Similar strategies and assumptions have led to the elaboration of useful interaction models for sandalwood (Chastrette et al., 1990; Pierre, 1991) and anisic notes (Pierre, 1991). 'Hydrogen bonding/dispersion forces' theory, which considers the pattern of interactions involved in the molecule-receptor site complex, offers a satisfactory explanatory and predictive model for several types of odors.

As it is based on the geometrical requirements of the interactions between molecules and receptor sites, this approach stresses the dissymmetry of molecule-site interactions rather than the molecular dissymmetry itself. Consequently, it seems specially efficient to deal with the role of chirality in structure-odor relationships.

Considering first the dispersion interactions, which exist between two hydrophobic parts of molecule, the only geometrical requirement is that the two interacting partners are not too far from each other (Reichardt, 1988). Therefore, there should be little or no chirality influence for compounds only interacting with the site by dispersion forces.

On the contrary, as hydrogen bonding requires a precise geometry, the odor of compounds interacting through hydrogen bonds should be very sensitive to absolute configuration, taking into account some variations due to molecular flexibility.

In so far as olfactory notes are related to a combination of interactions through hydrogen bonding and through dispersion forces, the spatial distribution of the interaction zones has to be considered. Slight differences in their disposition, altering the quality of the fit of the molecule with the site, should cause differences in olfactory perceptions. In any case, the odor of enantiomers should differ if their interactions with the sites are different, i.e. if their chiral parts are involved in the interactions.

The present paper explores the possibility of accounting for chiral aspects of odorant recognition assuming that dispersion interactions and hydrogen bonding are the most important factors.

\section{Materials and methods}

Structures and odors of 16 enantiomeric pairs well described in the literature were used (Table I and references within). For each compound, the authors compared odors of the two enantiomers in the same conditions. Therefore, we assume that the qualitative differences are significant and are not subjective impressions due to different concentrations in the samples.

Following the procedure previously described (Chastrette et al., 1990), comparisons between enantiomers were based on superimposition of lowest energy conformations of enantiomers and suitable reference molecules.

\section{Reference molecules}

Two enantiomers with the same olfactory notes are assumed to interact with the same 


No. Compound

1 Camphor

2 Isoborneol

3 2-Methyl-isobomeol

4 2-Methyl-borneol

5 Androstenone

6 Androsta-4,16-dien-one

7 2-Methyl-4-(5,5,6-exo-trimethyl-2exo-norbornyl)-cyclohexanone

8 Ambrox

9 9-Epiambrox

10 Isoambrox
Quality

Int. $\quad$ References

(+) Camphoraceous

(-) Camphoraceous

(+) Camphoraceous, celluloïd and rosemary oil note

(-) Camphoraceous, sweet and musty

(+) Camphoraceous, rubbery

(-) Camphoraceous, rubbery

(+) Camphoraceous, rubbery

(-) Camphoraceous, rubbery

(+) Urinous

(-) Odorless

(t) Unnous

(-) Odorless

(+) Urinous

(-) Urinous

(+) Woody, exotuc, spicy

(-) Exotic woody note and strong warm animal tonality

(+) Unknown

(-) Similar to (-)-Ambrox odor

- Ohloff et al. (1985)

(+) Considerable deviation from (-)-Ambrox odor

(-) Unknown
- Theimer et al. (1977)

1 Guillot and Babin (1949); Guillot and Thibaut (1951):

2 Guillot (1955)

- Tyler et al. (1978)

- Tyler el al. (1978)

$1000^{\mathrm{b}}$ Theimer et al. (1977);

0 Ohloff et al. (1983a)

1000 Ohioff et al (1983a,b)

0

200 Theimer et al. (1977)

10

100 Ohloff et al. (1985)

173

I Ohloff et al. (1985)
10 Escher et al. (1990); 
11 8,13-Epoxy-14,15, 16-trinorlabdane

12 Ambergris ketal

13 Nootkatone

14

Tricyclonootkatone

$15 \alpha$-Vetivone

16 Tetrahydronootkatone
(+) Woody

(-) Woody ambergris. musky and balsamic

(+) Woody ambergris, musky and balsamic

(-) Woody

$(+)$ Fresh and green and sour, woody and dusty

(-) Woody and dusty, spicy

(+) Woody and dusty, spicy

(-) Woody and dusty, fresh and green and sour

(t) Woody and dusty, fresh and green and sour

(-) Woody and dusty, spicy

(t) Woody and dusty, fresh and green and sour

(-) Woody and dusty, spicy weak

strong

strong Ohloff et al. (1980) weak

750 Haring et al. (1972)

1 Haring et al. (1972)

1.6

1 Haring et al. (1972)

22

$1 \quad$ Haring el al. (1972) 6.5

${ }^{D}$ Relative intensity of the two enantiomers in a pair.

${ }^{b}$ Arbitrary value. $(+) 5$ is the reference molecule for all urinous compounds in Table I

${ }^{c}$ Arbitrary value. $(-) 8$ is the reference molecule for the odor intensity of $(+) 8$ and enantiomers of compounds 9 and 10 
receptor site(s), and their intensity differences should be related to the greater capacity of interaction of one enantiomer. On the contrary, two enantiomers with different odor qualities cannot be assumed to interact with the same set of receptor sites. Consequently, chiral, rigid and stereochemically well-defined molecules of substances considered by perfumers as typical of the fragrance under consideration (i.e. showing all the characteristics of the odorant note with a strong intensity) were used in the structural comparisons, as reference molecules. It was assumed that the molecular structure of the reference molecule corresponds to a strong molecule-site interaction.

When no reference molecule was available, comparisons between enantiomers were based on computer-assisted superimposition of enantiomers from different pairs possessing the same note.

\section{Determination of low energy conformations}

Comparisons were based on the most stable conformation of each compound under study. This conformation was determined using the MMP2 program (Allinger, 1985), and taking into account the lone pairs of oxygen atoms. Considering that conformations in the vicinity of the receptor may differ from conformations in the gas phase, some conformations within $1 \mathrm{kcal} / \mathrm{mol}$ from the lower energy conformation were also considered occasionally.

\section{Determination of pertinent structural elements}

Pertinent structural elements are those atoms or groups of atoms which play a role in the interaction either through dispersion forces or through hydrogen bonding. They were determined on the basis of published works on structure-odor relationships.

To deal with hydrogen bonding we find it useful to consider as an atom of the molecule a fictive hydrogen atom hydrogen-bonded to an oxygen atom. This was achieved by replacing one of the lone pairs of the oxygen atom by a hydrogen bond, respecting the known geometrical constraints of hydrogen bonds to oxygen atoms (distances and angles).

\section{Comparison of models of molecules}

Comparisons were made with the Alchemy II software (1988) using a least-squares procedure to match only pairs of atoms involved in the interaction with the receptor site.

To match dispersion zones, atoms or groups of atoms assumed to be effectively involved in interaction with the receptor site(s) (e.g. methyl groups for the hydrophobic parts of the molecule) were matched. To compare hydrogen bonds, their orientation differences had to be considered.

Generally, superimpositions were made by matching carbon atoms involved in hydrophobic interactions and hydrogen atoms involved in hydrogen bonds.

The quality of the comparison was assessed using two measures of the quality of fit. The classical measure, included in the Alchemy II package, is an RMS parameter (root mean square distance) obtained from the distances between the paired atoms (a minimum of three pairs is required, but four pairs of atoms were used in all cases):

$$
\mathrm{RMS}=\left[{\left.\left.\underset{i=1}{\left(\sum_{i}\right.} d_{i}^{2}\right) / n\right]^{1 / 2}}^{n}\right.
$$


where $d_{i}$ is the distance between two paired atoms and $n$ is the number of pairs. RMS should have as low a value as possible, but as it is not necessary for the hydrophobic part of the molecule to completely fill the hydrophobic part of the pattern (Chastrette et al., 1990), it may vary on a relatively large scale.

Then we consider the angle $\alpha$ between the hydrogen bond of the reference molecule and the hydrogen bond of the molecule under comparison. Limits on the direction of hydrogen bonds being + or $-15^{\circ}$ (Legon and Millen, 1982; Murray-Rust and Glusker, 1984; Vedani and Dunitz, 1985) the angle $\alpha$ between the two hydrogen bonds of the two molecules under superimposition must be less than $30^{\circ}$ for similar interactions with the site.

The quality of fit was assessed on the basis of RMS and $\alpha$ values, with a priority to RMS.

A fit with RMS $<0.4$ and $\alpha<30^{\circ}$ was considered as a good fit. The limits correspond to a mean distance of $0.1 \AA$ between paired atoms and to the observed variability in hydrogen bond angles.

A fit with RMS $>1$ was always taken as a bad fit.

A fit with RMS between 0.4 and 1 was considered as acceptable provided $\alpha<30^{\circ}$. In the case where $\alpha>30^{\circ}$ the flexibility of the molecules must be considered. If $\alpha$ becomes $<30^{\circ}$ when using conformations other than the low energy conformation (with $\Delta \mathrm{E}<1 \mathrm{kcal}$ ), the fit was considered as a medium fit. All other cases correspond to a bad fit.

\section{Results and discussion}

In a preliminary unpublished study, careful examination of numerous data showed that enantiomeric pairs may be classified into three categories according to their odor differences.

(i) In category 1, the odors of the two enantiomers differ neither in intensity nor in quality (e.g. camphor and several camphoraceous compounds).

(ii) In category 2, enantiomers present the same main note but differ in secondary notes and in intensity [e.g. 2-methyl-4-(5,5,6-exo-trimethyl-2-exo-norbornyl)-cyclohexanone].

(iii) In category 3 , the odors of the two enantiomers differ both in quality and in intensity (e.g. nootkatone).

Any explanation of the role of chirality should account for the distribution of enantiomeric pairs into the above categories.

\section{Camphoraceous odorants}

The enantiomers of camphor 1 (Figure 1 and Table I) have substantially the same odor (Theimer et al., 1977). According to Beets' theory the enantiomers of this rigid polar monofunctional molecule, should exhibit a medium range difference in odor. Using our approach, as long as the ketone function is assumed to be involved in the interaction with the receptor site, either by its dipole or by hydrogen bonding, enantiomers of camphor 1 are not superimposable (RMS $=1.184, \alpha=45^{\circ}$ ) and should, therefore, have different odors. The oxygen atoms of the carbonyle function and the carbon atoms 


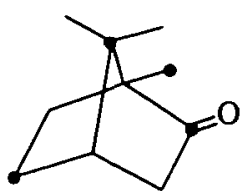

1

camphor

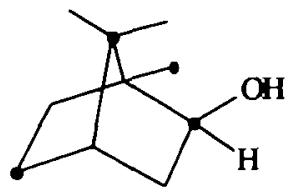

2

isoborneol

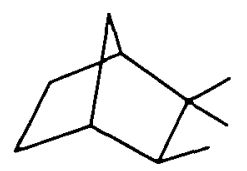

17

isocamphane

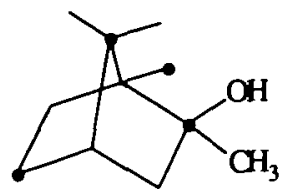

3

2-methyl-isoborneol

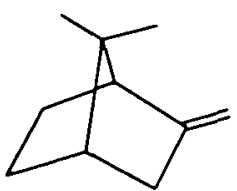

18

camphen

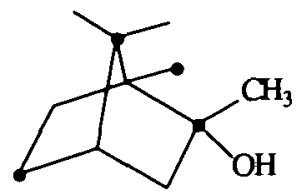

4

2-methyl-borneol

Fig. 1. Camphoraceous compounds.

labeled - in Figure 1 were used in the superimposition.

However the camphoraceous odor of hydrocarbons such as isocamphan 17 or camphen 18 (Figure 1) shows that the carbonyle function is not involved. It has been shown previously that camphoraceous molecules must have both a roughly spherical shape (Amoore, 1962) and a suitable volume, beween 90 and $180 \mathrm{~cm}^{3} \mathrm{~mol}^{-1}$ (Eminet and Chastrette, 1983). Assuming such a model, orientated interactions are not likely and chirality should have no influence on the camphoraceous note.

For all known enantiomeric pairs having the camphoraceous note, as principal or as secondary note, such as camphor 1 (Theimer et al., 1977), isoborneol 2 (Guillot and Babin, 1949; Guillot and Thibaut, 1951; Guillot, 1955), 2-methyl isoborneol 3 and 2-methyl borneol 4 (Tyler et al., 1978), chirality does not influence their olfactory characteristics (Table I).

Superimpositions of enantiomers of the above mentioned pairs on ( + )-Camphor $(+) 1$ by fitting the carbon atoms labeled - in Figure 1 led to a good quality of fit; all RMS were lower than 0.045 .

\section{Urinous odorants}

Both rigid polar monofunctional steroids and conformationally flexible polar monofunctional exo-norbornyl-cyclohexanone derivatives exhibit a urinous odor. The odor of steroids is highly influenced by their absolute configuration: in most cases only one enantiomer has this odor whereas the other one is odorless, e.g. compounds 5 and 6 , (Figure 2; Ohloff et al., 1983a,b). On the contrary enantiomers of the exo-norbornylcyclohexanone type compound (Figure 2), are not so different: they show the same odor quality, but differ in intensity (Theimer et al., 1977).

$d$-Androstenone $(+) 5$ is considered by perfumers as the reference compound for this odor. In previous works (Beets and Theimer, 1970; Theimer et al., 1977; Ohloff et al. , 1983b) it has been pointed out that a molecule exhibits a urinous odor if it possesses an oxygen atom and a $t$-butyl group (or an equivalent group), respectively, superimposable on the oxygen atom and the quaternary carbon atom No. 13 of $d$-androstenone (+)5 considered as the center of the hydrophobic part (Figure 2). 


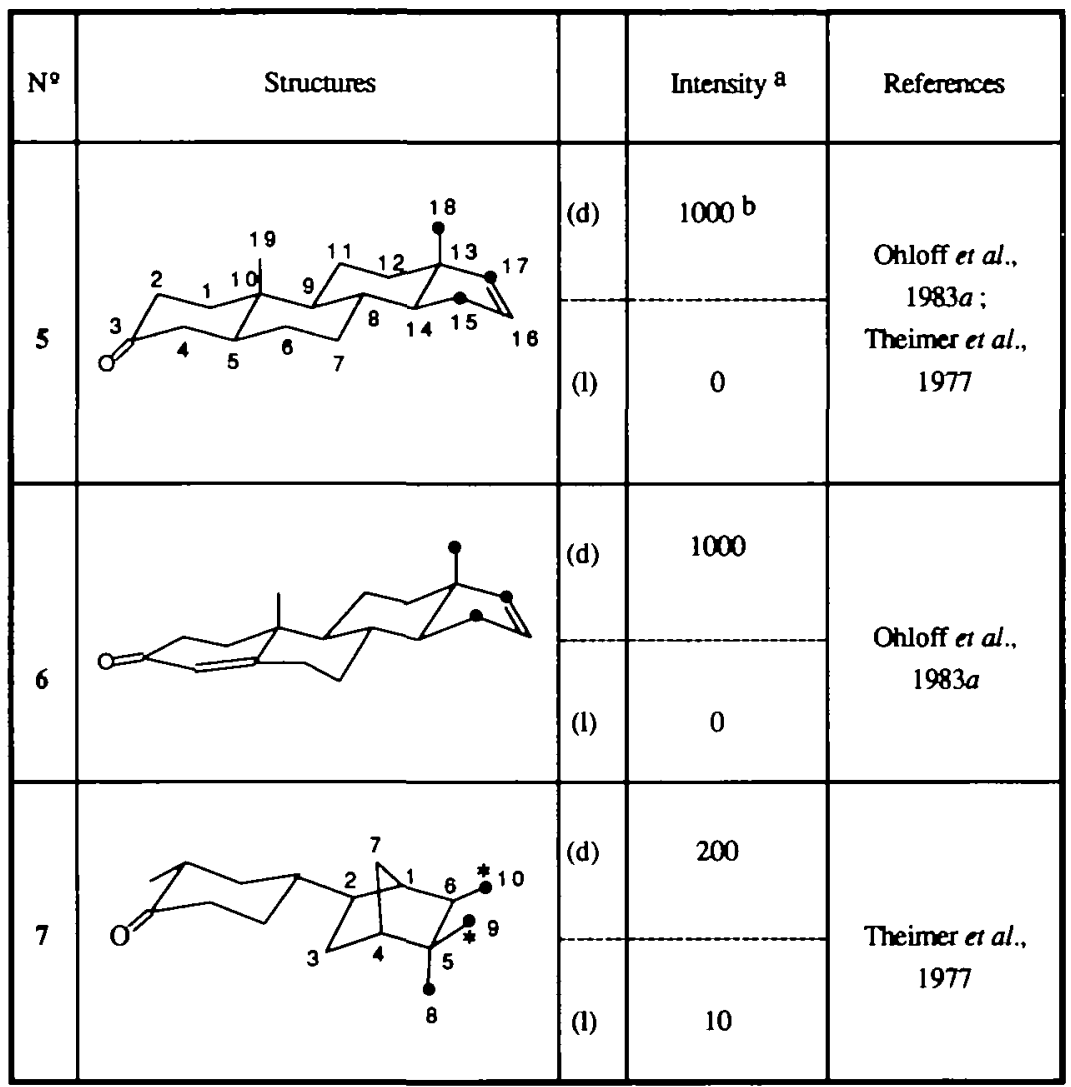

Fig. 2. Role of chirality on the urinous odor. (a) Relative intensity of the urinous odor of the two enantiomers in a pair. (b) According to perfumers, $d$-androstenone $(+) 5$ exhibits a powerful and characteristic urinous odor. Theimer et al. (1977), propose 1000 as arbitrary intensity value for this compound, and estimate the relative intensity of the other urinous compounds compared with it.

Table II. Superimposition with (d)-Androstenone (+)5

\begin{tabular}{lcc}
\hline No. & RMS & $\alpha$ \\
\hline$(-) 5$ & 1.204 & $61.8^{\circ}$ \\
$(+) 6$ & 0.041 & $2^{\circ}$ \\
$(-) 6$ & 1.196 & $61.6^{\circ}$ \\
$(+) 7$ & 0.327 & $6.5^{\circ}$ \\
$(-) 7$ & 1.073 & $10^{\circ}$ \\
& $0.223^{\circ}$ & $15^{\circ}$ \\
\hline
\end{tabular}

"Superimposituon involving the fictive hydrogen atom of the hydrogen-bond and only two carbon atoms.

In a first approach, and considering the preliminary results, carbonyle functions and quaternary carbon atoms of (-)5, and enantiomers of compounds 6 and 7 were superimposed on the carbonyle function and the quaternary carbon atom No. 13 of $d$-androstenone $(+) 5$. These superimpositions were not helpful to explain the role of chirality for this odor: all enantiomers are well superimposed on the reference compound 
$(+) 5$ (RMS lower than 0.11 and $\alpha<4.5^{\circ}$ ). However, they showed that the hydrophobic part is larger and includes the carbon atom No. 18 of the axial methyl group and carbon atoms No. 15, 16 and 17 of the cyclohexene part of $(+) 5$.

Then, superimpositions were made on these new bases. For $d$-androstenone $(+) 5$, a hydrogen atom bonded to the oxygen atom and the carbon atoms No. 15, 17 and 18 of $(+) 5$ were considered. Superimpositions of $(-) 5$ and enantiomers of compounds 6 and 7 were made by matching the hydrogen atom of the hydrogen-bond and the three carbon atoms labeled $\bullet$ or $\bullet *$ in Figure 2.

(i) Rigid 1-androstenone (-)5 (Figure 2) which is odorless (Ohloff et al., 1983a,b) fits very poorly with $(+) 5,\left(\right.$ RMS $=1.204, a=61.8^{\circ}$; Table II).

(ii) The urinous dextrorotatory $(+) 6$ accurately fits with $(+) 5$ (RMS $=0.041$ and $\alpha=2^{\circ}$ ), while the odorless (-)6 fits very badly, (RMS $=1.196$ and $\alpha=54^{\circ}$; Table II).

(iii) For exo-norbornyl-cyclohexanone derivative 7 (Figure 2), the good fit of $(+) 7$ with $(+) 5\left(\mathrm{RMS}=0.327, \alpha=6.5^{\circ}\right)$ contrasts with the poor fit of the 20 times weaker $(-) 7\left(\mathrm{RMS}=1.073, \alpha=10^{\circ}\right)$.

The superimposition results for $(+) 7$ are consistent with its urinous odor. However, in the case of $(-) 7$, this enantiomer should have no urinous character at all. Hydrogen bonds of $(-) 7$ and $(+) 5$ have similar directions $\left(\alpha=10^{\circ}\right)$, but their hydrophobic parts are very badly fitted. Analysis of the superimposition of these two molecular structures shows that the axial methyl group No. 18 of $(+) 5$ and the methyl group No. 8 of $(-) 7$ (Figure 2) are put $3 \dot{A}$ apart and on both sides of the steroid skeleton.

The positions of these two structural elements being very different, we assume that the methyl group No. 8 of $(-) 7$ is out of the hydrophobic part of the urinous pattern and is not involved in the interaction. The fit becomes better (RMS $=0.223, \alpha=15^{\circ}$ ) (Table II) if we use, in the superimposition, only the hydrogen atom of the hydrogenbond and the two carbon atoms labeled $\bullet *$, excluding the axial methyl groups No. 18 of $(+) 5$ and No. 8 of $(-) 7$ (Figure 2). The fact that $(-) 7$ has a urinous odor 20 times weaker than $(+) 7$ could be related to a less efficient interaction with the receptor site (one hydrogen bond pole and two groups in the hydrophobic pole) than in the case of $(+) 7$ (one hydrogen bond pole and three groups in the hydrophobic pole).

Comparisons of more or less odorous enantiomers of three rigid or flexible compounds enable us to determine the indispensable structural parts involved in the production of the urinous odor. Consequently, a common pattern as well as an interaction model for the urinous odor involving first a hydrogen bond accepted by the carbonyle function, and second a dispersion interaction involving carbon atom Nos 13, 14, 15, 16, 17 and 18 , within a geometrical relation similar to that found in the reference molecule $d$-androstenone $(+) 5$ can be proposed (Figure 3 ).

\section{Ambergris odorants}

The ambergris note is a difficult case due to a peculiar complexity. In fact six different facets have been recognized in the amber note, namely 'wet mossy forest soil; strong tobacco; balsamic, sandalwood-like; warm animal tonality of musk; seaweed ocean; fecal' (Ohloff, 1969b). 


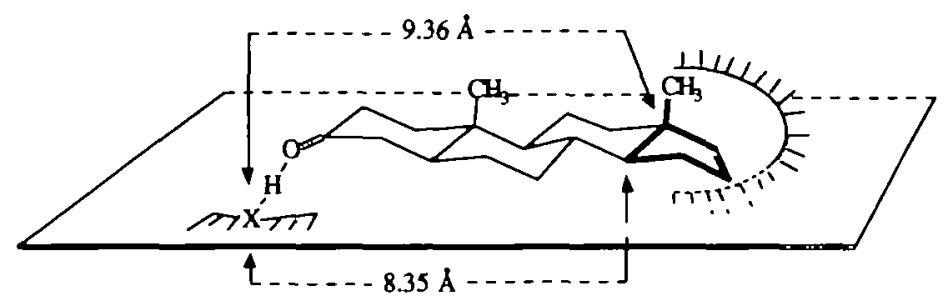

Fig. 3. Tentative interaction model for urinous odorants.

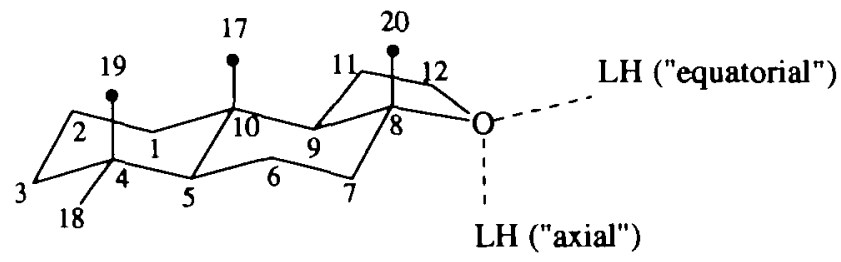

Fig. 4. Hydrogen bonds to (-)-Ambrox

However, '(-)-Ambrox (-)8 is recognized as prototype of all ambergris odorants both structurally and organoleptically' (Escher et al., 1990). In order to study the role of the chirality on the compounds with ambergris odor, $(-) 8$ was chosen as the reference compound in superimpositions.

To relate the ambergris note to molecular structure, the triaxial rule (Ohloff, 1971, 1982; Ohloff et al., 1976), and rules based on electronic properties and geometrical/structural features (Bersuker et al., 1985) or on the oxygen accessibility surface (Winter, 1989a,b) have been proposed, but no interaction model is known.

On the basis of the triaxial rule an oxygen atom and three axial methyl groups in 17, 19 and 20-positions labeled - in Figure 4 are assumed to be important interaction sites. Taking into account interaction through hydrogen bonding we based our superimpositions on four pairs of atoms: the hydrogen atoms hydrogen-bonded to oxygen atoms and the carbon atoms of three axial methyl groups (positions 17, 19 and 20). In fact, the oxygen atom can accept two hydrogen bonds through its two lone-pairs. In (-)-Ambrox (-)8 the first hydrogen-bond which is approximately in an 'equatorial position' is above the plane of the molecule, whereas the second one is in an 'axial position' but below the plane (Figure 4). These two hydrogen bonds having very different directions, we should be able to determine the hydrogen bond which is involved in the interaction by superimposing the two enantiomers of Ambrox 8 .

According to Ohloff et al. (1985), Ambrox (+)8 'with its higher threshold value $(2.4 \mathrm{ppb})$ and accentuated woody note lacks the strong and warm animal note of its enantiomer. (t)-Ambrox has, therefore, been called poor man's ambrox by our perfumers. The exotic, spicy undertone in (+)-Ambrox disappear in its racemate, for which a threshold concentration of $0.5 \mathrm{ppb}$ was measured'. So we consider that although $(+) 8$ shows some characteristics of the amber odor, it should not be taken as a true amber odorant.

Indeed, a very poor fit ( $\left.\mathrm{RMS}=0.615, \alpha=102.3^{\circ}\right)$ is obtained in the superimposition of $(+)$ and $(-)$-Ambrox 8 when the three axial methyl groups and the "equatorial 
hydrogen-bond' hydrogen atoms are matched. In this superimposition $(+) 8$ and $(-) 8$ are not even in the same plane and should exhibit completely different odors.

A bad fit (RMS $=1.315, \alpha=10.5^{\circ}$ ) is also obtained when the hydrogen atoms of the 'axial hydrogen-bond' are considered. The two enantiomers are roughly in the same plane and the assumed interaction sites are well superimposed, except for the methyl groups in position 17 which are put $2 \AA$ apart. According to the fact that $(+) 8$ and $(-) 8$ have different but nevertheless similar odors, the methyl group in position 17 appears to be necessary to have the true amber note, whereas its absence does not impede the presence of some facets of the ambergris odor.

These first results suggest that compounds can exhibit an amber odor if their molecules contain three methyl groups and an 'axial hydrogen bond' within a geometrical relation similar to that found in (-)-Ambrox (-)8. Even though only one enantiomer is known for 9-epiambrox 9 and Isoambrox 10 (Figure 5), both of which are epimers of $(-)$-Ambrox $(-) 8$, their superimpositions with $(-) 8$ confirm this hypothesis on the interactions.

The good fit of $(-) 9$ with $(-) 8$ (RMS $\left.=0.303, \alpha=25.5^{\circ}\right)$ shows that they can interact with the same receptor site.

In accordance with its odor profile which shows a considerable deviation from the model compound $(-) 8$ (Ohloff $e t$ al., 1985), (+)-Isoambrox (+) 10 fits very badly with (-)-Ambrox (-)8 (RMS $=2.165, \alpha=22^{\circ}$ ).

Assuming an interaction model, the role of chirality on the amber odor of compounds 11 and 12 (Figure 5) can be explained by superimposing their molecular structures with the three axial methyl groups No. 17, 19 and 20 labeled $\bullet$ and the hydrogen atom of 'axial hydrogen bond' of (-)-Ambrox (-)8 (Figure 4).

The levorotatory tricyclic compound ( -$) 11$ (Figure 5) is described as woody, amber, musky and balsamic, whereas its antipode $(+) 11$ is only weakly woody (Ohloff $e t$ al., 1980 ). In agreement with their odor descriptions, $(-) 11$ shows a good fit with $(-) 8$ RMS $=0.073, \alpha=8.1^{\circ}$ ), while the $d$-isomer fits very poorly $(\mathrm{RMS}=1.333$, $\left.\alpha=18.3^{\circ}\right)$.

The $l$-isomer fits well with $(-) 8$ using an 'axial hydrogen bond' $(\mathrm{RMS}=0.073$, $\alpha=8.1^{\circ}$ ) in agreement with the fact that its odor is described as woody, amber. The $d$-isomer which is weakly woody fits very poorly (RMS $=1.333, \alpha=18.3^{\circ}$ ).

The $d$-isomer of Ambergris Ketal (+)12 (Figure 5) is described as having a strong amber odor (Dey and Wolf, 1978; Brunke, 1980) or alternatively as 'woody ambergris like in the sense of ambrox' (Ohloff et al., 1980). Its enantiomer (-)12 (Figure 5) is described as weakly woody (Dey and Wolf, 1978; Ohloff et al., 1980; Brunke, 1989).

If we assume that the equatorial oxygen atom No. 16 (Figure 5) interacts with the receptor site, $(+) 12$ shows a good fit with $(-) 8\left(\mathrm{RMS}=0.176, \alpha=26.9^{\circ}\right)$ and the $l$-isomer (-)12 fits very poorly with (-)-Ambrox (-)8 (RMS $=1.351, \alpha=19^{\circ}$ ).

\section{Nootkatone type compounds (grapefruit and woody)}

While the $l$-isomer $(-) 13$ of nootkatone (Figure 6 ) is mainly woody with a very weak grapefruit background note, the $d$-isomer $(+) 13$ has a strong main grapefruit note with a woody undernote (Haring et al., 1972; Figure 7).

Interestingly, small structural modifications of 13 (cyclisation, hydrogenation, shift or addition of double bonds) lead to compounds such as tricycloketone $14, \alpha$-vetivone 


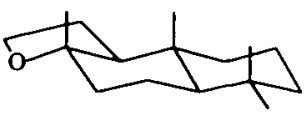

$(+) 8$

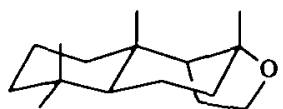

$(-) 9$

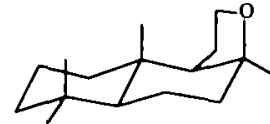

$(+) 10$

(+)-Ambrox

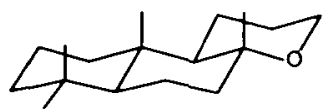

(-) 11

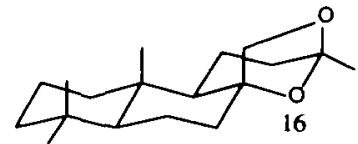

(t) 12

Fig. 5. Structure-ambergris note relationshıps

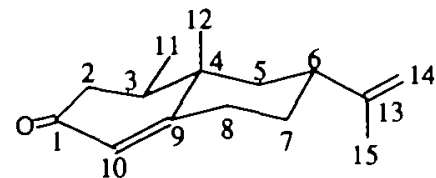

(+) 13

(+)-nootkatone

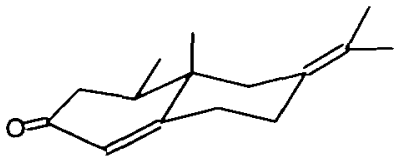

(+) 15

$(+)-\alpha$-vetivone

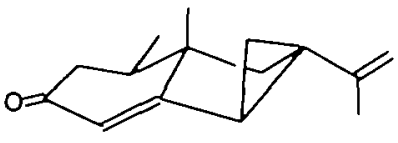

$(-) 14$

(-)-tricyclonootkatone

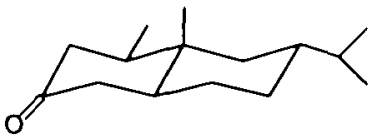

(+) 16

(+)-tetrahydronootkatone

Fig. 6. Nootkatone derivatives.

15 and tetrahydronootkatone 16 (Figure 6) whose enantiomers differ only by their secondary notes. Their main note, the woody one, is weakly sensitive to chirality, while their secondary notes differ in intensity (Haring et al., 1972). Enantiomers $(-) 14,(+) 15$ and $(+) 16$ have a noticeable grapefruit secondary note (Figure 7) while enantiomers $(+) 14,(-) 15$ and $(-) 16$ have only a very weak grapefruit note.

No interaction models are known for grapefruit and woody notes, and because of its complex odor, nootkatone $(+) 13$ cannot be considered as the reference molecule for the grapefruit note. However it can be expected that the enantiomers $(-) 14,(+) 15$ and $(+) 16$, which may be called 'grapefruit enantiomers' because of their noticeable secondary note, will fit with nootkatone $(+) 13$, while the enantiomers $(+) 14,(-) 15$ and $(-) 16$, considered as 'woody enantiomers' should fit with nootkatone $(-) 13$ rather accurately. Following an approach used in earlier examples, we assume that all these compounds interact with the receptor sites by hydrogen bonds and dispersion forces. To compare their molecular structures, the enantiomers of compounds 14,15 and 16 have been superimposed with $(+)$ and $(-)$ nootkatone which are, respectively, the most representative structures of grapefruit and woody notes in this sample. These superimpositions have been made by matching the hydrogen atom hydrogen-bonded to the 
nooktatone 13

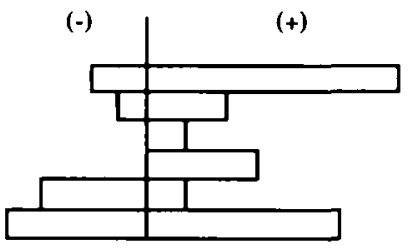

tricycloketone 14

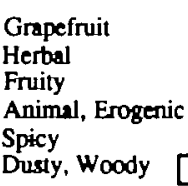

(.)

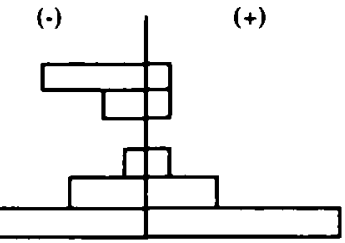

$\alpha$-véturone 15

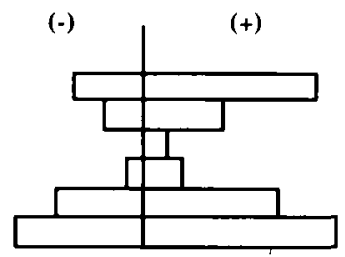

tetrahydronootkatone 16

(-)

$(+)$
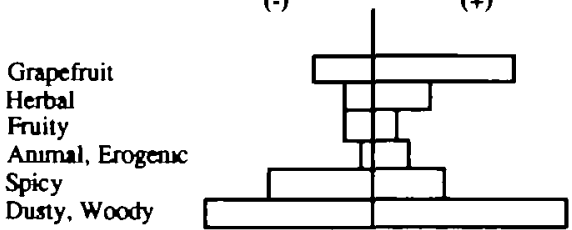

Fig. 7. Odor profiles of nootkatone and its denvatives after Hanng et al. (1972).

oxygen atom of the carbonyle function and carbon atoms No. 12, 14 and 15 of (+) and (-)-nootkatone (Figure 5) with the corresponding atoms of the enantiomers of compounds 14,15 and 16 .

Fitting Nootkatone $(+) 13$ and the 'grapefruit enantiomers' we found RMS values between 0.084 and 0.543 , and $\alpha$ angles lower than $18.9^{\circ}[(-)$-tricycloketone $(-) 14$ which shows the weakest grapefruit note among the 'grapefruit enantiomers', has the highest RMS and $\alpha$ values $\left(0.543 ; 18.9^{\circ}\right)$ ]. The same values were obtained by fitting nootkatone (-)13 and the 'woody enantiomers'. One enantiomer from the first series does not fit well with any enantiomer from the second series (RMS between 0.017 and 0.543 , and $\alpha$ values between $74.2^{\circ}$ and $85.1^{\circ}$ ).

This suggests a possible use of these enantiomeric pairs in the determination of the grapefruit and woody interaction models, although none of them can be considered as a reference for these odors and although these notes are likely to correspond to more than one interaction pattern between molecules and receptor sites. We assume that the common structural parts of the eight enantiomers are somehow important for the woody note which they all possess, while the structural parts which are found only in the 'grapefruit enantiomers' and (+)13 have to be related to the grapefruit note. In order to test these assumptions we are currently comparing other molecules with the same grapefruit or woody notes, but different chemical structures.

\section{General discussion}

To explain odor differences of similarities within pairs of enantiomers by comparison of their molecular structures one has to determine first what molecular characteristics should be used and then how to assess their similarity.

Beets stressed the importance of global molecular characteristics such as dipoles and functionalities. Odors predicted by this theory were not found in agreement with observed odors for several pairs of enantiomers corresponding to rigid polar monofunctional or 
bifunctional molecules. We have obtained a better agreement using a process based on superimpositions restricted to selected molecular parts which actually interact with the receptor site. While this approach seems theoretically sound its practical use calls for some caution.

Two steps are crucial:

(i) The proper selection of interacting parts or binding sites which relies on empirical rules or in a few cases on existing models.

(ii) Once the superimposition is made, the assessment of the quality of fit and determination of limits between good, acceptable and bad fits. An empirical approach based on statistics being ruled out by the small number of available enantiomeric pairs, the choice of limits was based on interaction energies.

Dispersion interactions are known to depend strongly on distances between interacting atoms and, consequently, two atoms in a matched pair cannot be expected to interact with the same site if the superimpositions puts them too far apart. To determine the acceptable variations in distances let us consider the simple case of dispersion forces between two hydrogen atoms.

The dispersion energy reaches a maximum when the two interacting hydrogen atoms are $2.4 \dot{A}$ apart. Using the Alchemy II force field it is calculated that increasing the distance between them to $2.5 \AA$ decreases the dispersion energy to $94 \%$ of its value. Increasing the distance to $2.6 \AA$ (i.e. a variation of $0.2 \AA$ instead of $0.1 \AA$ ) decreases the dispersion energy to $81 \%$ of its value. However, decreasing the distance to 2.28 $\dot{A}$ and $2.16 \AA$ decreases the interaction energy, respectively, to 87 and $22 \%$ of its optimal value.

Taking into account both kinds of variations we considered a priori that a good fit corresponds to a variation of about $10 \%$ in interaction energies and a variation of less than $0.1 \AA$ in distances. The results obtained on several examples show that the good fits are well within this limit.

As in previous studies, we observed here that molecules can act as olfactory stimuli even when they interact with only a part of the hydrophobic zone of the receptor. In such cases it was found that the olfactory note was present but with a weak intensity. No attempt was made to determine the acceptable limits of occupation of a receptor site.

Hydrogen bonds are sensitive to both distances and directions. As the nature of the hydrogen bond donor in the site is unknown, a mean distance of $1.8 \dot{\mathrm{A}}$ was chosen. It is known from crystallographic studies that hydrogen bond directions are distributed around a maximum of probability in a relatively narrow band with a width of about $30^{\circ}$ (Legon and Millen, 1982; Murray-Rust and Glusker, 1984; Vedani and Dunitz, 1985). It was assumed that the energy of hydrogen bonds outside this band was too small to contribute to the interaction. Consequently, all fits with $\alpha>30^{\circ}$ were considered bad except when it was possible to find other conformations of the molecule (within a range of $1 \mathrm{kcal} / \mathrm{mol}$ ) leading to $\alpha$ values smaller than $30^{\circ}$. This is a rather strict rule as differences of $1 \mathrm{kcal} / \mathrm{mol}$ between conformations are frequently observed.

In this paper models and empirical rules were used to explain olfactory properties of enantiomers. The relationships can be used in the opposite direction: odors of enantiomers are used to design interaction models. This approach led to a tentative 
model for the urinous odor and opened some ways towards a model for more complex odors such as the ambergris odor. The limits here are that an olfactory note does not necessarily correspond to a single receptor site: the interactions between a population of structurally and configurationally identical stimulant molecules and a population of receptor sites of a chemoreceptory system generate an informational pattern which can be described with a series of olfactory notes (Beets, 1978a,b). However, it was possible to put forward some assumptions on models for the grapefruit and woody notes found in the complex scent of the nootkatone and its derivatives.

\section{Acknowledgements}

The authors gratefully thank the Fondation Roudnitska, for its financial support, and G.Sicard for helpful discussions.

\section{References}

Alchemy II (1988) Molecular modeling softure. Tripos Associates, Inc., Evans \& Sutherland, St Louis, MO, USA.

Allinger, N.L.(1972) Quantitative compansons of molecular shape and iterative site orientations. In Maxwell,R.A. (ed.), Pharmacology and the Future of Man, Proc. Int. Congr. Pharmacol. 5th, (Pub. 1973), 5, 57-63. C.A.: $8067642 \mathrm{f}$.

Allinger,L. (1985) MMP2 program. Indiana University, USA.

Amoore,J.E. (1962) The stereochemical theory of olfaction. Elucidation of the stereochemical properties of the olfactory receptor sites. Proc. Sci. Sect. Toilet Goods Ass., Suppl. to 37, 13-23.

Amoore,J.E. (1963a) Stereochemical theory of olfaction. Nature, 198, 271-272.

Amoore,J.E. (1963b) Stereochemical theory of olfaction. Nature, 199, 912-913.

Amoore,J.E. (1964) Current status of the steric theory of odour. Ann. N.Y. Acad. Sci., 116, 457-476.

Amoore,J.E. (1971) Stereochemical and vibrational theones of odour. Nature, 233, 270-271.

Beets,M.G.J. (1978a) (ed.) Structure-Activity Relationships in Human Chemoreception. Applied Science Publishers, London, pp. 29-82.

Beets, M.G.J. (1978b) (ed.) Structure-Activity Relatıonships in Human Chemoreception. Applied Science Publishers L.T.D., London, pp. 149- 188.

Beets,M.G.J. (1978c) (ed.) Structure-Activity Relationships in Human Chemoreception. Applied Sctence Publishers L.T.D., London, pp. 127-148.

Beets,M.G.J. and Theimer,E.T. (1970) Odour similarity between structurally unrelated odorants. In Wolstenholme,G.E.W., Knight,J. and Churchill,A. (eds), Ciba Foundation Symposium on Taste and Smell in Vertebrates. Ciba, London, pp. 313-323.

Bersuker,I.B., Dimoglo,A.S., Gorbachov,M.Yu., Koltsa,M.N. and Vlad,P.F. (1985) Structural and electronic ongin of ambergris ador of cyclic compounds. Nouv. J. de chimie, 9, 211-217.

Brunke,E.J. (1980) Lower homologues and analogues of ambergris fragrance compounds. In Van der Stare,M. (ed.), Olfaction and Taste VII. IRL Press Ltd., London and Washington, pp. 31-34.

Buck,L. and Axel,R. (1991) A novel multigene family may encode odorant receptors: a molecular basis for odor recognition. Cell, 65, 175-187.

Chastrette,M. (1990) Stimulus properties and binding to receptors. In Schild,D.H. (ed.), NATO ASI Series, Chemosensory information processing, 39, pp. 97-107.

Chastrette,M. and Zakarya,D. (1988) Sur le rôle de la liaison hydrogène dans l'interaction entre les recepteurs olfactifs et les molécules à odeur de musc. C. $R$. Acad. Sci. Paris, 307(II), 1185-1188.

Chastrette,M., Zakarya,D. and Pierre,C. (1990) Relations structure-odeur de bois de santal: Recherche d'un modzle d'interaction basé sur le concept d'hypermotif santalophore. Eur. J. Med. Chem., $24,433-440$.

Dey,A.K. and Wolf,H.R. (1978) Über die Herstellung von äther der enantio-14,15-dinorlabdan-reihe aus eperusäure. Helv Chim. Acta, 61, $1004-1010$.

Eminet,B.P. and Chastrette,M. (1983) Discrimination of camptroraceous substances using physico-chemical parameters. Chem. Senses, 7, 293-300.

Escher,S., Giersch,W., Niclass, Y., Bernardinelli,G. and Ohloff,G. (1990) Configuration-odor relationship in SB-Ambrox. Helv Chim. Acta, 73, 1935-1947. 
Friedman,L. and Miller,J.G. (1971) Odor incongruity and chirality. Science, 172, 1044-1046.

Guillot,M. (1955) Pouvoir rotatoire et olfaction. Recherches, 1955 (mai), 24-31.

Guillot,M and Babin,R. (1949) Pouvoir rotatoire et odeur. C.R. Acad. Sci. Paris, 229(2), 1363-1365.

Guillot,M. and Thubaut,P. (1951) Influence du pouvoir rotatoire sur la qualité de l'odeur. C.R. Acad. Sci. Paris, 232(1), $1138-1140$.

Hansch,C. and Leo,A.J. (1979) Substituent Constants for Correlation Analysis in Chemistry and Biology, Wiley, New York.

Hansch,C., Muir,R.M., Fujita,T., Maloney,P.P., Geiger,F. and Sreich,D.C. (1963) The correlation of biological acitvity of plant growth regulators and chloromycetin denvatives with Hammet constants and partition coefficient. J. Am. Chem. Sac., 85, 2817-2824.

Haring,H.G., Rijkens,F., Boelens,H. and van det Gen,A. (1972) Olfactory studies on enantiomeric eremophilanes sesquiterpenoids. J. Agric. Good Chem., 20, 1018-1021.

Jacobs,F.N. and Elliot,D. (1975) Individual and substance differences in the discriminality of optrcal isomers. Chem. Senses Flavor, 1, 317-321.

Legon, A.C. and Millen,D.J. (1982) Determination of properties of hydrogen-bonded dimers by rotational spectroscopy and a classification of dimer geometries. Faraday Dicuss. Chem. Soc., 73, 71-87.

Mosandl,A. and Günther,C. (1989) Stereoisomeric flavor compounds. 20. Structure and properties of $\gamma$-lactone enantiomers. J. Agric. Food Chem., 37, 413-418.

Mosandl,A. and Heusinger,G. (1985) 1,3-oxathianes, chiral frut flavor compounds. Liebigs Ann. Chem., $1185-1191$.

Mosandl,A., Hener, U., Hagenauer-Herner, U and Kustermann, A. (1990) Stereoisomeric flavor compounds. 33. Multidimentional gas chromatography direct enantiomer separation of $\gamma$-lactones from fruits, foods and beverages. J. Agric. Food Chem., 38, 767-771.

Murray-Rust,P. and Glusker,J.P. (1984) Directional hydrogene bonding to $\mathrm{sp}^{2}$ and $\mathrm{sp}^{3}$ hybridized oxygen atoms and its relevance to ligand-macromolecule interactions. J. Am. Chem. Soc., 106, 1018-1025.

Mutschler,Von E., Lambrecht,G. and Moser,U. (1986) Chirale Wirkstoffe als Werkzeuge zur Subklassifizierung Pharmakologischer Rezeptoren. Disch. Apoth. Ztg., 126, 2012-2019.

Ohloff,G. (1969a) Odorous properties of enantiomeric compounds. In Pfaffmann,C. (ed.), Olfaction and Taste IV. Rockfeller University Press, New York, pp. 156-160.

Ohloff,G. (1969b) Chemie der Geruchs und Geschmackstoffe. Fortschr. Chem. Forsch., 12, 185-251.

Ohloff,G. (1971) Relationship between odor sensation and stereochemistry of decalin ring compounds. In Gustation and olfaction, an International Symposium, Geneva, June 1970. Aademic Press, London, New York, pp. $178-188$.

Ohloff,G. (1982) Structure-activity relationships in ambergris odorants. In Theimer,E.T. (ed.), Fragrance Chemistry. Academıc Press, New York, pp. 535-573.

Ohloff,G. (1986) Chemistry of odor stımuli. Experientia, 42, 271-279.

Ohloff,G., Giersch, W., Schulte-Elte,K.H. and Vial,C. (1976) Zur Stereochemue der Geruchswahrnehmung von 1-dekalon-derivaten und ihren Oxaanalogen Verbindungen. Helv Chim. Acta, 59, 1140-1157

Ohloff,G., Vial,C., Wolf,H.R., Job,K., Jegou,E., Polonski,J. and Lederer,E. (1980) Stereochemistry-ador relationships in enantiomeric ambergris fragrances. Helv Chim. Acta, 63, 1932- 1946.

Ohloff,G., Maurer, B., Winter,B. and Giersch,W. (1983a) Structural and configurational dependence of the sensory process in steroids. Helv Chim. Acta, 66, 192-217.

Ohloff,G., Giersch,W., Thommen,W. and Willhalm,B. (1983b) Conformationally controlled odor perception in 'steroid-type' scent molecules. Helv Chim. Acta, 66, 1343-1354.

Ohloff,G., Giersch,W., Pickenhagen,W., Furrer,A. and Frei,B. (1985) Significance of the geminal dimethyl group in odor principle of Ambrox ${ }^{\circ}$. Helv Chim. Acta, 68, 2022-2029.

Pickenhagen,W. and Brönner-Schindler,H. (1984) Enantioselective synthesis of (+)- and (-)-cis-2-methyl4-propyl-1,3-oxathiane and their olfactive properies. Helv Chim. Acta, 67, 947-952.

Pierre,C. (1991) Contriburtion à l'etude des composes anists et santalés: Relations structure-odeur et syntheses. Universite Claude Bernard, Lyon I, Thze de doctorat, No. 11691.

Pike,M.L., Melvin,P.E. and Homung,D.E. (1988) Quality and intensity differences of enantiomers when tested separately and in mixtures. Chem. Senses, 13, 307-309.

Polak,E.H., Fombom,A.M., Tilquin,C. and Punter,P.H. (1989) Sensory evidence for olfactory receptors with opposite chiral selectivity. Behav. Brain Res., 31, 199-206.

Reichard,C. (od.) (1988) Sotvents and Solvent Effects in Organic Chemistry, 2nd odn. VCH Verlagsgesellschaft Weinheim (Federal Republic of Germany), 5-41.

Revial,M.F., Sicard,G., Duchamp,A. and Holley,A. (1982) New studies on odour discrimination in the frog's olfactory receptor cells. I. Experimental results. Chem. Senses, 7, 175-190. 


\section{M.Chastrette et al.}

Revial,M.F., Sicard,G., Duchamp,A. and Holley,A. (1983) New studies on odour discrimination in the frog's olfactory receptor cells. II. Mathematical analysis of electrophysiological responses. Chem. Senses, 8, $179-194$.

Schallenberger,R.S. and Acree,T.E. (1971) Chemical structure of compound and their sweet and bitter taste. In Beidler, L.M. (ed.), Handboak of Sensory Phyisology. Chemical Senses and Taste. Springer, New York, IV(2), pp. $221-277$

Silversteın,R.M. (1978) Chemical Ecology: Odor Communicatıon in Animals. In Riıtter,F J. (ed.), Proc. Adv. Res. Inst. 1978. (Pub. 1979), Elsevier, Amsterdam, pp. 133-153.

Testa,B. (1986) Chiral aspects of drug metabolism. Trends Pharmacol. ScI., 7, 60-64

Theimer,E.T., Yoshida,T. and Klaıber,E.M. (1977) Olfaction and molecular shape. Chirality as a requisite for odor. J. Agric. Food Chem., 25, 1168-1177.

Tyler,D., Acree,T.E. and Butts,R.M (1978) Odor characterization of the synthetic stereossomers of 2-methylborneol. J. Agric. Food Chem., 26, 1415-1417

Vedani,A. and Dunitz.J.D. (1985) Lone-pair directionality in hydrogen bond potentual functions for molecular mechanics calculations: The inhibition of human carbonic anhydrase II by sulfonamides. J. Am. Chem. Soc., 107, 7653-7658.

Winter,B. (1989a) Ring-opened analogues of Ambrox ${ }^{\star}$ : synthesis and structure-odour relationships. Helv. Chim. Acta, 72, 1278-1283.

Winter,B. (1989b) QSAR in olfaction. ambergris-type odorants In Fauchère,J.L and Liss,A.R. (eds), QSAR: Quantitative structure-Activity Relationships in Drug Design. New York, pp. 401-405.

Zakarya,D. (1988) Recherche de relations structure-odeur de musc. Mise au point d'une nouvelle méthode G.E.S.D.E.M. Université Claude Bernard, Lyon I, These de doctorat, No. 0288.

Recetved on April 22, 1992; accepted on May 29, 1992 